British Journal of Nutrition (2021), 126, 1237-1246

doi:10.1017/S0007114520005139

(C) The Author(s), 2020. Published by Cambridge University Press on behalf of The Nutrition Society. This is an Open Access article, distributed under the terms of the Creative Commons Attribution licence (http://creativecommons.org/licenses/by/4.0/), which permits unrestricted re-use, distribution, and reproduction in any medium, provided the original work is properly cited.

\title{
Dietary patterns and trajectories of global- and domain-specific cognitive decline in the Lothian Birth Cohort 1936
}

\author{
Janie Corley* and Ian J. Deary \\ Lothian Birth Cohorts Group, Department of Psychology, University of Edinburgh, Edinburgh, UK \\ (Submitted 3 August 2020 - Final revision received 3 December 2020 - Accepted 15 December 2020 - First published online 23 December 2020 )
}

Abstract

Healthy dietary patterns may protect against age-related cognitive decline, but results of studies have been inconsistent and few have had extensive longitudinal follow-up with comprehensive cognitive testing. The aim of the present study was to determine associations of dietary patterns with trajectories of global- and domain-specific cognitive change over a 12-year period. Data from 863 community-dwelling, dementia-free participants from the Lothian Birth Cohort 1936 study of ageing completed a FFQ at baseline (aged 70 years) and underwent cognitive testing at baseline, and at the ages of 73, 76,79 and 82 years. Composite cognitive scores were constructed for four cognitive domains (visuospatial ability, processing speed, memory and verbal ability) and global cognitive function. A Mediterranean-style pattern and a traditional pattern were derived using principal component analysis of self-reported dietary intakes. In fully adjusted latent growth curve models, higher baseline adherence to the Mediterranean-style dietary pattern $(\beta=0.056, P=0.009)$ and lower baseline adherence to the traditional dietary pattern ( $\beta=-0.087, P<0.001$ ) were cross-sectionally associated with better verbal ability. A slightly steeper decline in verbal ability over 12 years was observed in those with higher Mediterranean-style diet scores at baseline ( $\beta=-0 \cdot 003, P=0 \cdot 008)$. All other associations were non-significant. Our findings in this well-characterised Scottish cohort indicate that adherence to a healthy Mediterranean-style diet is associated crosssectionally with better verbal (crystallised) ability, with the converse being true for the traditional diet. A healthier baseline diet did not predict a reduced risk of global- or domain-specific cognitive decline.

\section{Key words: Dietary patterns: Cognitive decline: Cohort studies: Older adults: Latent growth modelling}

Cognitive decline is the progressive deterioration in cognitive functions including some aspects of memory, speed of processing and some reasoning abilities and may lead to dementia ${ }^{(1,2)}$. Diet has been suggested to play an important role in healthy brain ageing $^{(3)}$. Adhering to particular dietary patterns may represent a viable strategy for reducing age-related cognitive decline and neurodegenerative pathologies such as Alzheimer's disease and other forms of dementia ${ }^{(4,5)}$. The Mediterranean diet emphasises plant foods and other dietary components reported to be neuroprotective and thus has received the most attention to date, with the majority of systematic reviews reporting beneficial outcomes on cognitive health ${ }^{(6-8)}$.

Many observational studies provide evidence for a 'protective' role of healthy dietary patterns, such as the Mediterranean diet, on cognitive ageing ${ }^{(9-14)}$. The Chicago Health and Aging Project ${ }^{(15)}$ and the Memory and Aging Project ${ }^{(16)}$ found that higher Mediterranean diet adherence was associated with slower rates of cognitive decline among 3790 older adults ( $\geq 65$ years) over a mean period of 7.6 years, and among 826 older adults $(81.5$ (sD 7.1) years) over a mean period of 4.1 years, respectively. In contrast, several longitudinal studies report no association between Mediterranean diet adherence and cognitive decline or mild cognitive impairment over time $^{(17-19)}$, while others report mixed findings. For example, the Australian Imaging Biomarkers and Lifestyle study of Ageing reported no significant associations in 527 adults (69 years), except for $A p o E$ e 4 allele carriers, in whom higher Mediterranean diet scores were associated with less 3-year decline in executive functioning, but not in other domains ${ }^{(20)}$

Conflicting evidence on the association between dietary patterns and later-life cognition may be due to methodological differences between studies, including sample age. Studies in older populations $>70$ years are relatively rare, especially those which present extensive longitudinal follow-up, hampering efforts to assess whether diet predicts trajectories of cognitive change across the eighth and ninth decades of life. Second, many (but not all) previous studies have assessed global cognitive function only ${ }^{(21)}$ often using a single measure of global cognitive status, such as the Mini-Mental State Examination (MMSE), which

Abbreviations: FDR, false discovery rate; IQ, intelligence quotient; LBC1936, Lothian Birth Cohort 1936; MMSE, Mini-Mental State Examination; PCA, principal component analysis.

* Corresponding author: Dr Janie Corley, email Janie.Corley@ed.ac.uk 
has low sensitivity for detecting sub-clinical age-related cognitive change. Examining domains of cognitive function such as memory, executive function and processing speed is important, as the effects of diet may not be consistent across domains, and different abilities may have unique patterns and mechanisms of decline.

The Lothian Birth Cohort 1936 (LBC1936) study previously demonstrated cross-sectional associations of better cognitive performance with closer adherence to a Mediterranean-style dietary pattern and lower adherence to a more traditional dietary pattern, at age 70 years $^{(22)}$. In the present study, we extend these results, using the same 'a posteriori' baseline dietary patterns, derived using principal component analysis (PCA) of 'wholediet' data, to predict 12-year cognitive change (age 70-82 years) in the same sample. To address heterogeneity in cognitive decline, we assess trajectories of global- and domain- specific cognitive change using an extensive battery of cognitive tests repeated over five waves of assessment. The LBC1936 study is a well-characterised cohort based in Scotland, for whom there are measures of cognitive ability from early life.

\section{Subjects and methods}

The Lothian Birth Cohort 1936 is a community-dwelling sample of 1091 individuals being studied in later life for the purposes of assessing the determinants of cognitive and brain ageing. All were born in 1936, and most are surviving participants of the Scottish Mental Survey of $1947^{(23)}$. At study entry (2004-2007, age 70 years, $n 1091)$ and subsequently every 3 years at ages 73 ( $n$ 866), 76 ( $n$ 697), $79(n 550)$ and 82 years ( $n$ 431), participants were invited to undergo repeated assessments for cognitive, health and psychosocial assessment. Full details on the background, recruitment and data collection procedures are available ${ }^{(24)}$.

A flow chart detailing how the analytic sample for the present study was derived is presented in online Supplementary Fig. S1. From the full quota of participants who satisfactorily completed a FFQ at age 70 years baseline ( $n$ 882), nineteen participants were excluded from our analyses because they had a history of dementia. The analytic sample for the present study comprised 863 individuals at age 70 years (baseline), 711 at age 73 years, 580 at age 76 years, 456 at age 79 years and 364 at age 82 years.

\section{Ethics}

This study was conducted according to the guidelines laid down in the Declaration of Helsinki, and all procedures involving human subjects were approved by the Multicentre Research Ethics Committee for Scotland (baseline, MREC/01/0/56), the Lothian Research Ethics Committee (baseline, LREC/2003/2/29) and the Scotland A Research Ethics Committee (age 73, 76, 79, 82 years, 07/MRE00/58). Written informed consent was obtained from all subjects.

\section{Assessment of dietary intake}

Dietary intake was assessed at baseline using a self-administered, semi-quantitative 168-item FFQ (Scottish Collaborative Group FFQ version 7.0) ${ }^{(25)}$ validated for use in community-dwelling older populations ${ }^{(26)}$. Participants indicated how frequently they consume particular foods and beverages by selecting one of nine possible responses ranging from 'rarely or never' to ' $7+$ measures per day'. A total of 967 participants (89\%) at baseline completed an FFQ. In line with standard operating procedures for the $\mathrm{FFQ}^{(25)}$, questionnaires with ten or more missing values ( $n 39)$ and those defined as having extreme energy intakes $(<2 \cdot 5$ th or $>95$ th centile of intake, $n$ 46) were excluded from the analysis.

\section{Principal component analysis}

The dietary patterns used in the current study were identified previously at baseline and reported first in Mõttus et al. ${ }^{(27)}$. In brief, PCA was used to extract dietary factors on the basis of correlation of all the individual FFQ items. Components were retained with eigenvalues $>1$, based on the examination of Scree plots. The retained factors were orthogonally rotated by the varimax method so that the factors were uncorrelated, making them easier to interpret. Each rotated component was interpreted based on the food items with loadings of $>0 \cdot 30$, which were considered as significantly contributing to a pattern. Factor scores were calculated for each component for each participant, by summing the frequency of consumption multiplied by factor loadings across all food items. Higher scores represent closer dietary adherence. The scores represent standardised variables with a mean of 0 and a standard deviation of 1 . Dietary pattern scores were adjusted for overall energy intake using the residual method ${ }^{(28)}$ at the time of calculation. As such, energy intake was not required to be included as a covariate in subsequent models.

The first component, characterised by consumption (i.e. factor loadings of $>0 \cdot 30$ ) of vegetables (leeks or courgettes, tomatoes, broccoli, spinach, other salad vegetables), fish, legumes, olive oil, salad dressing, poultry, pasta, rice, water and tomato-based sauces, was labelled a 'Mediterranean-style' dietary pattern. A second component, characterised by greater consumption (i.e. factor loadings $>0.30$ ) of meat, processed meats (such as pies, pasties and sausage rolls), mashed potatoes, tinned vegetables, peas or beans, carrots, baked beans, bottled sauces, custard or other sweet sauces, milk-based puddings (and a negative loading from filter, espresso and cappuccino coffee denoting lower consumption), was consistent with a traditional Scottish convenience diet and labelled a 'traditional' dietary pattern. Online Supplementary Table S1 lists the food items with factor loadings $>0.30$ on each of these two patterns.

\section{Assessment of cognitive function}

A comprehensive neuropsychological battery of well-validated tests was administered at each wave by trained psychologists, in accordance with standard protocols, using the same location and equipment. The LBC1936 cognitive test battery has been described in detail previously in an open-access protocol article ${ }^{(24)}$. Most of the tests derive from the Wechsler Adult Intelligence Scale III-UK edition (WAIS-III $\left.^{\mathrm{UK}}\right)^{(29)}$ and the Wechsler Memory Scale III-UK edition $\left(\text { WMS-III }{ }^{\mathrm{UK}}\right)^{(30)}$. Specifically, we examined four cognitive domains: visuospatial ability (Matrix Reasoning (WAIS-III ${ }^{\mathrm{UK}}$ ), Block Design (WAISIII $^{\mathrm{UK}}$ ), Spatial Span (WMS-III ${ }^{\mathrm{UK}}$ ), average of Forwards and Backwards); processing speed (Digit Symbol (WAIS-III ${ }^{\mathrm{UK}}$ ), Symbol 
Search (WAIS-III ${ }^{\mathrm{UK}}$ ), Choice Reaction Time ${ }^{(31)}$, Inspection Time ${ }^{(32)}$; memory (Logical Memory Immediate and Delayed Recall (WMSIII ${ }^{\mathrm{UK}}$ ), Verbal Pairs Immediate and Delayed Recall (WMS$\left.\mathrm{III}^{\mathrm{UK}}\right)$, Digits Backwards (WAIS-III $\left.{ }^{\mathrm{UK}}\right)$ ); and verbal ability (National Adult Reading Test ${ }^{(33)}$, Wechsler Test of Adult Reading ${ }^{(34)}$ and Verbal Fluency ${ }^{(35)}$ ).

The scores for each cognitive test were standardised to $z$-scores using baseline mean and standard deviation values. Subsequently, $z$-scores of individual cognitive tests were averaged to produce domain $z$-scores in visuospatial ability, processing speed, memory and verbal ability. A composite cognitive score reflecting global cognitive function was calculated as an average of the thirteen individual cognitive tests' $z$-scores. Higher scores indicate better performance (reaction time scores were converted to indicate lower scores with slower reaction times). Decision on grouping of cognitive tests was based on a previously defined correlational structure in the LBC1936 test battery ${ }^{(36,37)}$.

\section{Covariates}

Risk factors known to be associated with cognitive ageing in this cohort were included ${ }^{(36,37)}$ and derived from baseline data. Demographic, lifestyle and health information were obtained by interview, physical examination, questionnaire and blood sampling. Education was coded as number of years in formal full-time education. Socio-economic status was derived from occupational social class which was coded according to highest achieved occupation using the Classification of Occupations, $1980^{(38)}$ into six categories ranging from 1 (highest professional occupations) to 5 (unskilled occupations), with 3 (skilled occupations) divided into $3 \mathrm{~N}$ (non-manual) and $3 \mathrm{M}$ (manual). Marital status comprised five categories (married, single, divorced, cohabiting, widowed). Childhood cognitive ability (age 11 years intelligence quotient (IQ) scores) was derived from Moray House Test ${ }^{(23)}$ scores taken by participants as part of the Scottish Mental Survey of 1947, at age 11 years. The Moray House Test is a group-administered, general intelligence/IQtype test, with a 45-min time limit. It comprises verbal reasoning, numerical and other items. In concurrent validity testing, it correlated about $r 0.8$ in childhood with the individually administered Stanford-Binet test ${ }^{(39)}$. Raw Moray House Test scores were corrected for age in days at time of testing and converted into standard IQ-type (continuous) scores for the sample, where mean is 100 and standard deviation is 15 . Age 11 years IQ is an important predictor of cognitive ability in later life (cognitive ability is highly stable across the life course until well into the ninth decade $\left.{ }^{(40)}\right)$ and a confounder of diet-cognition associations in adulthood in the $\mathrm{LBC} 1936^{(22)}$. Smoking history was categorised as never, former or current smoking. Physical activity level was derived a lifestyle questionnaire and ranged from level 1 ('moving only in accordance with household chores') to level 6 ('keep fit or aerobic exercise several times a week'). BMI was calculated using height and weight measurements taken by trained nurses at the time of assessment. History of disease was coded dichotomously (yes/no) for hypertension, diabetes and CVD. ApoE e 4 carrier status was derived from whole blood samples taken on the day of baseline assessment by trained nurses. Genomic DNA was isolated from whole blood, and the target sequences for two polymorphic sites (rs7412 and rs429358) were genotyped with TaqMan technology by the Wellcome Trust Clinical Research Facility Genetics Core, Western General Hospital, Edinburgh.

\section{Statistical analysis}

Participants were divided into tertiles of dietary patterns for descriptive and illustration purposes only. Background characteristics according to dietary adherence tertiles were compared using $\chi^{2}$ or ANOVA as appropriate. The full continua of scores were used in the main analyses for maximum statistical power.

We used latent growth curve models, a structural equation modelling technique, to analyse level (i.e. latent intercept, age 70 years) and trajectory of change (i.e. latent slope, age 70-82 years) in cognitive function across five waves of testing simultaneously, in relation to baseline diet (which was assessed on a continuous scale) and baseline covariates. Models were fitted to each cognitive outcome measure: global cognitive function; visuospatial ability; processing speed; memory and verbal ability. Thus, for these five composite cognitive variables, we modelled a level (age 70 years baseline cognitive function) and a linear slope (from age 70 to 82 years), taking all five cognitive measurements into account. The slope of cognitive function represents the rate at which cognitive functions change over subsequent waves of testing, otherwise known as relative cognitive decline. In our models, we used the average time lag between the waves: 2.98 years, waves $1-2 ; 6.75$ years, waves $1-3 ; 9.81$ years, waves $1-4$; and 12.53 years, waves $1-5$, as the path weights for calculation of the slope factor. The path from the slope factor to the initial wave's test score was set to zero. In the latent growth curve models, the continuous distribution of diet adherence scores was used as the predictor variable. Model 1 adjusted for age and sex. Model 2 additionally adjusted for age 11 years IQ. Model 3 additionally adjusted for age 11 years IQ, ApoE e 4 status, smoking status, physical activity, marital status and socio-economic status. Models were run independently for each dietary pattern. A structural diagram to illustrate these measured and latent variables is presented in Fig. 1.

Model coefficients were estimated using full-information maximum likelihood estimation to take all data into account, including individuals who did not complete all waves of testing. Model fit was tested using four indices of absolute fit: root-mean-square error of approximation with values $<0.06$ considered acceptable; comparative fit index with values $>0.95$ considered acceptable and standardised root-mean-square residual with values $<0.08$ considered acceptable. We report unstandardised estimates and standard errors. The false discovery rate (FDR) correction for multiple testing was applied to the resultant $P$ values. All statistical analyses were performed using R version 3.6.1 'Action of the Toes' (R Foundation for Statistical Computing) and the latent variable analysis package 'lavaan' ${ }^{\text {(41) }}$ for growth curve modelling.

\section{Results}

\section{Descriptive statistics}

Demographic characteristics of the study population at baseline stratified by tertiles of dietary adherence (low, moderate, high) 


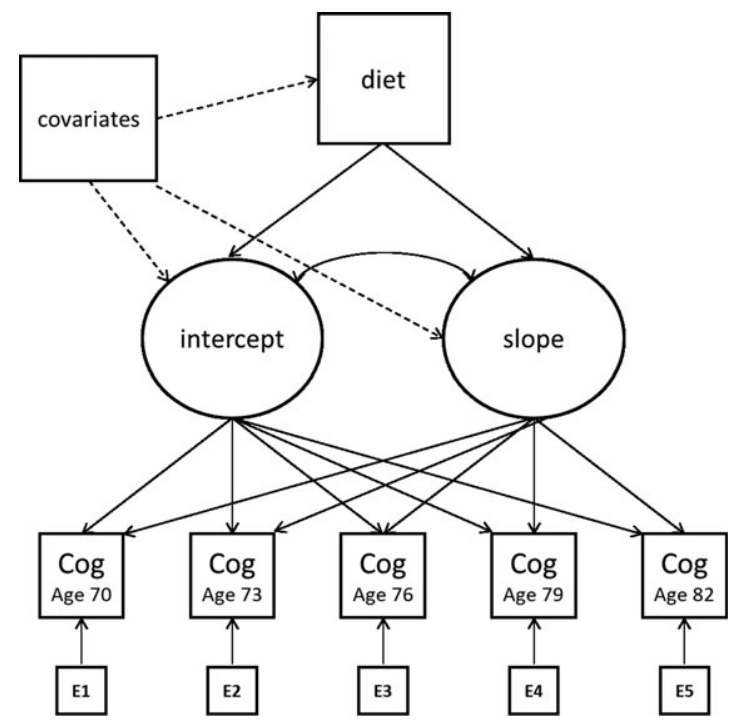

Fig. 1. A simplified path diagram of the latent growth curve model of cognitive function and dietary patterns. Circles represent latent variables, and squares represent measured variables. Growth curves, including a latent intercept and slope factor, were calculated for each of the five composite cognitive variables using individual cognitive test data across five time points. The slope of cognition represents change in this outcome over 12 years of follow-up. The regressions of diet (predictor) on cognitive intercept and slope were the associations of interest. For clarity, this figure represents one cognitive domain only. Dashed lines represent the relationships between covariates that were fixed across waves. We tested three different covariate models for predicting cognitive intercept and slope: model 1 (age + sex); model 2 (model $1+$ age 11 years intelligence quotient); model 3 (model $2+A p o E$ e4, smoking, physical activity, marital status, socio-economic status).

are presented in Table 1 . The study sample comprised 863 participants $(50.2 \%$ male) with an average age of 69.5 (SD 0.8 ) years at baseline, henceforth referred to as age 70 years, and followed-up for a mean of 12.5 (SD 0.5) years. Closer adherence to the Mediterranean-style dietary pattern was associated with lower age $(P=0.008)$, more education, higher age 11 years IQ, higher socio-economic status, more physical activity (all $P<0.001$ ) and less smoking $(P=0.003)$. Closer adherence to the traditional dietary pattern was associated with being male, less education, a lower age 11 years IQ (all $P<0.001)$, more smoking $(P=0.003)$, less physical activity $(P=0.015)$ and higher BMI $(P=0.001)$. Associations with history of disease were not significant. Correlations between age 11 years IQ and dietary pattern scores were $r 0.17(P<0.001)$ and $r-0.23(P<0.001)$ for the Mediterranean-style and traditional dietary patterns, respectively. Childhood IQ and years of education correlated $r 0.40(P<0 \cdot 001)$.

The demographic characteristics of the sample by completer status is presented in online Supplementary Table S2. Those who remained in the study to final follow up (completers, $n$ 364) had higher childhood IQ scores, more education, higher socioeconomic status, higher activity levels, lower BMI, less smoking and less likely to have hypertension or diabetes, than those who did not ( $n$ 499). Raw cognitive scores for each test (organised by cognitive domain) at each wave of the study, for this sample, are presented in online Supplementary Table S3. Mean composite cognitive $z$-scores (for global cognitive function, visuospatial ability, processing speed, memory and verbal ability) at baseline (wave 1) and the end of the follow-up period (wave 5) are presented in online Supplementary Table S4 for the full sample ( $n$ 863) and for completers only ( $n$ 364). Participants who went on to complete all waves of the study were more likely to have higher cognitive scores at baseline compared with participants who did not remain to follow up. Within the subgroup of completers, participants showed the greatest cognitive decline in tasks requiring processing speed and visuospatial ability. Verbal ability mean scores were marginally higher at wave $5($ mean $=0 \cdot 20(\mathrm{SD} 0 \cdot 8)$ ) compared with wave $1($ mean $=0.19(\mathrm{SD} 0 \cdot 7)$ ) for completers

To illustrate cognitive decline across domains, we plotted global cognitive function and domain scores across all five

Table 1. Participant characteristics by tertile of dietary pattern adherence in the Lothian Birth Cohort 1936 study* (Mean values and standard deviations; percentages)

\begin{tabular}{|c|c|c|c|c|c|c|c|c|c|c|c|c|c|c|}
\hline \multirow[b]{3}{*}{ Characteristics } & \multicolumn{7}{|c|}{ Mediterranean diet score } & \multicolumn{7}{|c|}{ Traditional diet score } \\
\hline & \multicolumn{2}{|c|}{ Low (n 287) } & \multicolumn{2}{|c|}{$\operatorname{Mod}(n 288)$} & \multicolumn{2}{|c|}{ High (n 288) } & \multirow[b]{2}{*}{$P_{\text {for trend }}$} & \multicolumn{2}{|c|}{ Low (n 287) } & \multicolumn{2}{|c|}{ Mod (n 288) } & \multicolumn{2}{|c|}{ High (n 288) } & \multirow[b]{2}{*}{$P_{\text {for trend }}$} \\
\hline & Mean & SD & Mean & SD & Mean & SD & & Mean & SD & Mean & SD & Mean & SD & \\
\hline Age (years) & $69 \cdot 6$ & 0.8 & 69.5 & 0.8 & $69 \cdot 4$ & 0.8 & 0.008 & $69 \cdot 3$ & 0.9 & $69 \cdot 6$ & 0.8 & $69 \cdot 6$ & 0.8 & 0.003 \\
\hline Education (years) & $10 \cdot 5$ & 0.9 & $10 \cdot 8$ & $1 \cdot 1$ & $11 \cdot 1$ & $1 \cdot 2$ & $<0.001$ & $11 \cdot 2$ & $1 \cdot 2$ & $10 \cdot 6$ & $1 \cdot 1$ & $10 \cdot 5$ & $1 \cdot 0$ & $<0.001$ \\
\hline Age 11 years IQ score† & 98.9 & $12 \cdot 9$ & $101 \cdot 7$ & $14 \cdot 7$ & $103 \cdot 7$ & 13.9 & $<0.001$ & $106 \cdot 3$ & $12 \cdot 7$ & $100 \cdot 3$ & $12 \cdot 89$ & $97 \cdot 7$ & $14 \cdot 7$ & $<0.001$ \\
\hline Physical activity level & $2 \cdot 8$ & $1 \cdot 1$ & 3.0 & $1 \cdot 1$ & 3.2 & $1 \cdot 1$ & $<0.001$ & $3 \cdot 1$ & $1 \cdot 1$ & 3.0 & $1 \cdot 1$ & 2.9 & 1.0 & 0.015 \\
\hline BMI $\left(\mathrm{kg} / \mathrm{m}^{2}\right)$ & $27 \cdot 7$ & 4.2 & 27.5 & 4.2 & $27 \cdot 4$ & 4.1 & 0.71 & $26 \cdot 8$ & $4 \cdot 1$ & $27 \cdot 6$ & $4 \cdot 1$ & $28 \cdot 2$ & 4.2 & 0.001 \\
\hline \multicolumn{15}{|l|}{ Categorical variables } \\
\hline Sex (male \%) & \multicolumn{2}{|c|}{$52 \cdot 3$} & \multicolumn{2}{|c|}{$48 \cdot 3$} & \multicolumn{2}{|c|}{$44 \cdot 4$} & 0.17 & \multicolumn{2}{|c|}{38.0} & \multicolumn{2}{|c|}{$49 \cdot 7$} & \multicolumn{2}{|c|}{$57 \cdot 3$} & $<0.001$ \\
\hline Marital status (married \%) & \multicolumn{2}{|c|}{$70 \cdot 0$} & \multicolumn{2}{|c|}{$75 \cdot 3$} & \multicolumn{2}{|c|}{$73 \cdot 6$} & 0.76 & \multicolumn{2}{|c|}{$70 \cdot 0$} & \multicolumn{2}{|c|}{74.7} & \multicolumn{2}{|c|}{$74 \cdot 3$} & 0.72 \\
\hline SES (professional \%) & \multicolumn{2}{|c|}{$70 \cdot 5$} & \multicolumn{2}{|c|}{$82 \cdot 6$} & \multicolumn{2}{|c|}{$89 \cdot 8$} & $<0.001$ & \multicolumn{2}{|c|}{$94 \cdot 0$} & \multicolumn{2}{|c|}{$78 \cdot 6$} & \multicolumn{2}{|c|}{$70 \cdot 0$} & $<0.001$ \\
\hline ApoE e4 (\% with allele) & \multicolumn{2}{|c|}{$24 \cdot 0$} & \multicolumn{2}{|c|}{29.8} & 31.0 & & 0.15 & 31. & & & & 26 & & 0.40 \\
\hline Smoking (current \%) & 14.6 & & 11 & & 4.9 & & 0.003 & 7. & & & 0 & 14 & & 0.003 \\
\hline Smoking (former \%) & $40 \cdot 8$ & & 40 & & $46 \cdot 5$ & & - & 40 . & & & & 45 & & - \\
\hline Smoking (never \%) & $44 \cdot 6$ & & 47 & & $48 \cdot 6$ & & - & 52 . & & & & 39. & & - \\
\hline Hypertension (\% yes) & $42 \cdot 2$ & & 39 & & $36 \cdot 1$ & & 0.34 & 36 & & & & 44. & & 0.09 \\
\hline Diabetes (\% yes) & 8.7 & & 7 & & 4.9 & & 0.18 & 5 & & & 3 & 8 & & 0.27 \\
\hline CVD (\% yes) & $24 \cdot 7$ & & 24 & & 23.3 & & 0.92 & 23. & & & & 27 . & & 0.31 \\
\hline
\end{tabular}

Mod, moderate; IQ, intelligence quotient; SES, socio-economic status.

* Means and standard deviations for continuous variables or percentages for categorical variables. $P$ from ANOVA or $\chi^{2}$ tests as appropriate.

† Age 11 years IQ (childhood cognitive ability) derived from scores on the Moray House Test completed during the Scottish Mental Survey of 1947. 
waves, according to tertiles of dietary pattern adherence at baseline, with individuals categorised as either belonging to a low adherence, moderate adherence or high adherence group (Fig. 2). Fig. 2 is based on data from completers only ( $n$ 364) as including data from all individuals in the sample would bias the plots towards better performance with increasing age.

\section{Dietary patterns and cognitive function (cross-sectional associations)}

Table 2 (left columns) presents the intercept associations of baseline dietary pattern scores with cognitive function level at age 70 years using latent growth curve model. Model fit statistics of all latent growth curve models indicated a good fit and ranged from: root-mean-square error of approximation 0.037-0.061; standardised root-mean-square residual 0.011-0.026; comparative fit index 0.958-0.992 (see online Supplementary Table S5 for all fit statistics). In these models, dietary pattern scores were treated as continuous variables. Unstandardised coefficients, standard errors and level of significance are reported. We tested an interaction term between $A p O E$ e 4 genotype and diet which was not significant for any of the cognitive domains; therefore, models were not further stratified by $A p O E$ carrier $v$. non-carrier status. In the basic model (with the fewest covariates, i.e. just age and sex), higher baseline adherence to the Mediterranean-style dietary pattern was significantly associated with better cognitive function, and higher baseline adherence to the traditional dietary pattern with lower cognitive function, across all four cognitive domains and global cognitive function, at age 70 years. The addition of childhood IQ to the basic model caused significant attenuation in effect sizes for the intercept by an average of $68 \%$ (Mediterranean-style pattern) and $65 \%$ (traditional pattern) across the cognitive domains. In the fully adjusted model, higher adherence to the Mediterranean-style pattern was associated with better verbal ability $(\beta=0.056$ (SE 0.021), $P=0.009)$ at age 70 years and higher adherence to the traditional diet pattern scores was associated with lower verbal ability ( $\beta=-0.087$ (sE 0.021 ), $P<0.001$ ), both of which were robust to FDR correction. All other cross-sectional (intercept) associations between diet and cognitive function were not FDR significant in the fully adjusted model.

\section{Dietary patterns and cognitive change (longitudinal associations)}

Table 2 (right columns) presents the longitudinal slope associations of baseline diet with cognitive change from age 70 to 82 years. We observed little evidence of associations of baseline diet with 12-year cognitive change, with the exception of a slightly steeper decline in verbal ability associated with higher Mediterranean-style diet adherence at baseline $(\beta=-0.003$ (sE 0.001), $P=0.008$ ), which remained significant following FDR correction. This finding was contrary to our hypotheses; however, we note that the change in this domain was minimal over time. Baseline adherence to a more traditional (less healthy) diet at age 70 years was not associated with 12-year cognitive change in global cognitive function or any of the domains measured. Online Supplementary Table S5 presents the slope associations for each variable included in the fully adjusted growth curve model of cognitive change for the Mediterranean-style and the traditional dietary patterns.

\section{Sensitivity analyses}

We conducted three sensitivity analyses, arising from the review process, to test the robustness of the cognitive intercept and slope. First, we additionally adjusted for three further potential health covariates in model 3, namely, BMI, CVD and diabetes. Second, we calculated a Mediterranean diet score ${ }^{(42)}$ and ran the growth curve models with Mediterranean diet score (range 0-9) as a predictor. Third, we ran the growth curve models without excluding participants with a history of dementia reported at any of the five waves of assessment. These additional analyses, presented in online Supplementary materials (online Supplementary Tables S6-S8), made little difference to the results reported above.

Finally, in post hoc analyses, we analysed the relative risk of low (1), moderate (2) and high (3) dietary pattern adherence status in relation to cognitive function and cognitive decline (data not presented). In line with the main analyses using the continuous measures, we found significant associations with baseline verbal ability only; no other associations were significant. The highest Mediterranean diet tertile was nominally associated with better verbal ability compared with the lowest tertile (estimate $=0 \cdot 179$, $95 \%$ CI $0 \cdot 01,0 \cdot 35, P=0 \cdot 04$, not FDR significant), but not compared with the middle tertile (estimate $=0 \cdot 149,95 \%$ CI $-0 \cdot 02$, $0 \cdot 32, P=0 \cdot 08$ ). Despite there being an overall (inverse) significant association between the traditional diet pattern tertiles and verbal ability, neither comparisons independently reached significance (low $v$. high: estimate $=0 \cdot 161,95 \%$ CI $-0 \cdot 001,0 \cdot 32, P=0.05$; moderate $v$. high: estimate $=0.078,95 \% \mathrm{CI}-0 \cdot 09,0 \cdot 25, P=0 \cdot 36$ ).

\section{Discussion}

We used five waves of data to examine trajectories of global- and domain-specific cognitive change in relation to two 'a posteriori' dietary patterns, among community-dwelling older adults in Scotland, who remained free from dementia over 12 years of follow-up. Higher adherence to a healthy, Mediterranean-style diet pattern, and lower adherence to a traditional (processed food) pattern, was associated cross-sectionally with better cognitive function at age 70 years across all of the cognitive measures, though only associations with verbal ability were significant after adjusting for childhood IQ and other covariates. Estimates from growth curve models show that the Mediterranean-style dietary pattern was not associated with 12-year change in global cognitive function, visuospatial ability, processing speed or memory domains. Contrary to our hypotheses, individuals with a higher Mediterranean-style diet score at baseline had a slightly steeper decline in verbal (crystallised) ability, though the effect size and overall change in this domain were small. Closer adherence to a more traditional diet pattern at baseline did not predict 12-year cognitive change across any of the domains measured. These findings are in line with and extend the results of a previous cross-sectional study in the LBC1936 at baseline ${ }^{(22)}$ and suggest that diet, assessed in a narrow age cohort of older Scottish adults, does not capture 

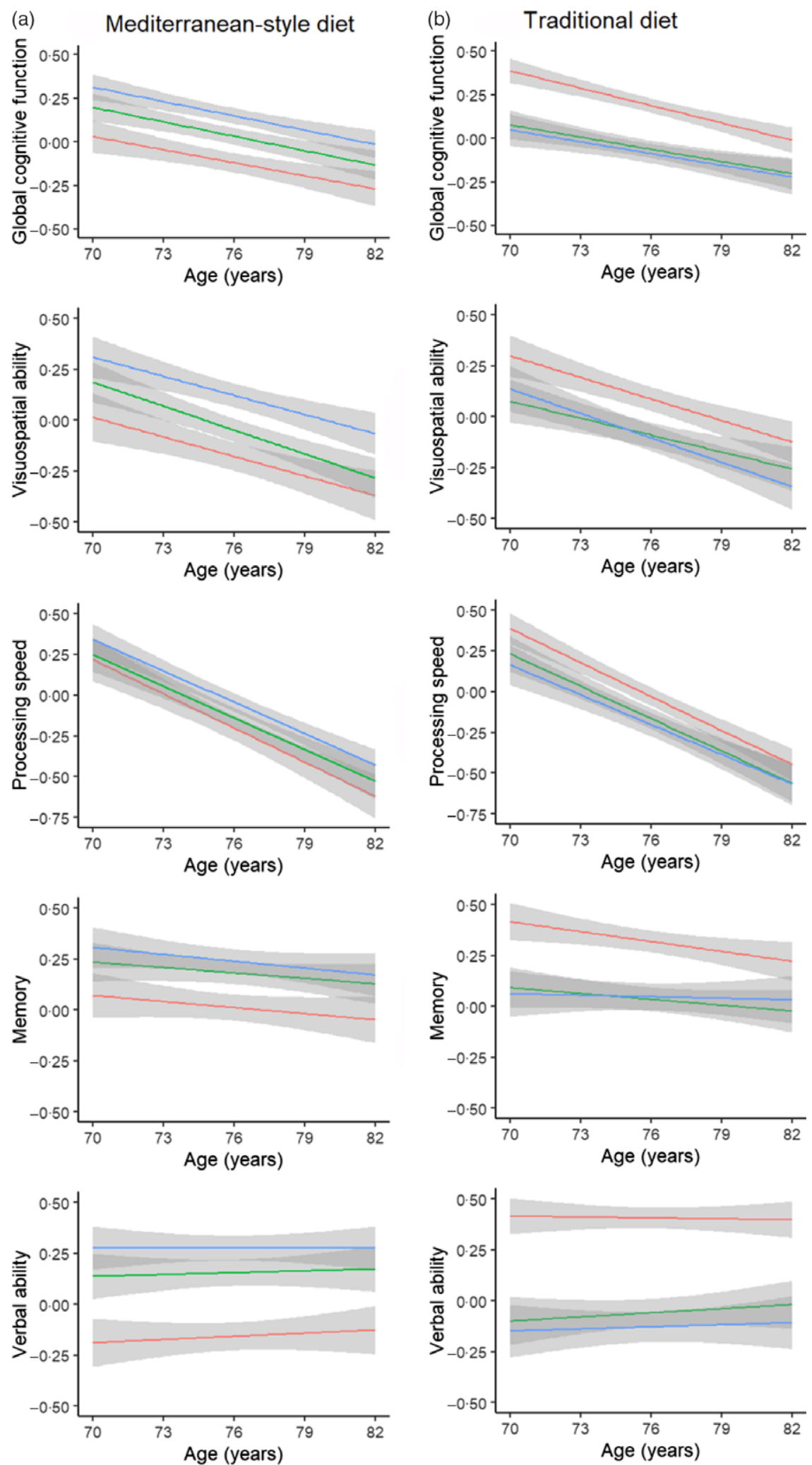

Fig. 2. Mean trajectories of global- and domain-specific cognitive performance using linear model fit lines stratified by baseline levels of dietary adherence score in the Lothian Birth Cohort 1936. (a) Mediterranean-style dietary pattern and (b) traditional dietary pattern. Stratification was by tertile category: low adherence; moderate adherence; high adherence. Grey bands indicate $95 \% \mathrm{Cl}$. These plots represent data from completers only, that is, individuals with data across all five assessments, since including the entire baseline sample would bias the means towards a positive direction with increasing age. Note that the main analyses in the report were done using the continua of adherence-to-diets scores and on all participants; these diet adherence tertiles on completers are for illustration only. - , High; - , moderate; - , low. 
significant variance in cognitive ageing trajectories, beyond other known predictors of cognitive change.

The finding that the Mediterranean-style diet was associated with steeper decline in verbal ability seems to contradict its positive relationship with cognition at baseline. However, the effect size was small, and likely to reflect regression to the mean ${ }^{(43)}$, that is, a consequence of higher ability individuals performing relatively more poorly on tests with known ceiling effects when followed longitudinally ${ }^{(44)}$. In simple terms, for well-performing individuals, the only way is down. In support of this theory, we note that previous studies have reported a similar pattern of findings for education; one study found that more education was associated with better baseline cognition, but each year of additional schooling increased rates of decline in global cognition and episodic memory by 0.004 and 0.012 standard score units, respectively ${ }^{(45)}$. Furthermore, this finding may not convey clinical importance for a domain that reflects learned vocabulary and word knowledge (otherwise known as crystallised intelligence) which, even in older individuals, is relatively stable with advancing age ${ }^{(46)}$

Nonetheless, our findings are broadly consistent with some previous longitudinal studies conducted exclusively in olderage samples, such as the Nurses' Health Study ( $n 16058$ women, $\geq 70$ years), in which adherence to the Mediterranean diet was associated with better cognition at baseline but not with a lower risk of 6-year cognitive decline ${ }^{(19)}$ and in the Women's Antioxidant Cardiovascular Study ( $n$ 2504, $\geq 65$ years), in which adherence to a Mediterranean-style diet was not associated with 5 -year cognitive decline ${ }^{(47)}$. In a UK study of much older individuals, The Newcastle $85+$ study ( $n 791,>85$ years), two PCAderived dietary patterns (healthy and less healthy) akin to the current study, was unrelated to cognitive change over a 5-year period $^{(48)}$. Similar findings are reported in two slightly younger cohorts: in the PATH Through Life study ( $n$ 1528, 60-64 years), a Mediterranean diet score was unrelated to global cognitive change over 4 years $^{(17)}$; and in the EPIC-Greece cohort $(n 732$, $>60$ years), a Mediterranean diet score was unrelated to change in MMSE score over 6-13 years ${ }^{(18)}$. Furthermore, a systematic review reported that most randomised controlled trials, which have the potential to identify causal associations, concluded that there was no significant association between the Mediterranean diet and reduction of cognitive decline, and very few showed a small effect size ${ }^{(49)}$.

Conversely, our results are in contrast to those of other longitudinal studies which support a protective effect of a Mediterranean diet on long-term cognitive health in older populations. Wengreen et al. reported a consistently higher level of global cognitive status over 11 years in adults $\geq 65$ years of the Cache County Study ${ }^{(14)}$. Two further prospective studies, the Chicago Health and Aging Project and the Memory and Aging project, have shown that adherence to Mediterranean-like dietary patterns was associated with slower global cognitive decline in adults $\geq 65$ years over a mean of 7 years ${ }^{(15)}$ and slower global and cognitive domain decline in adults aged 81.5 (sD $7 \cdot 1$ ) years over a mean of 4 years of follow-up ${ }^{(16)}$. The traditional diet pattern was not linked with a faster rate of cognitive decline in the current study, though it is high in saturated fat, sugar and processed foods. This finding is in contrast with other 
studies that report steeper cognitive decline with increasing adherence to a meat-based, Western dietary pattern, similar to the traditional pattern in the LBC1936, and with infrequent consumption of plant-based foods ${ }^{(13,20)}$. One potential reason is that there is less dietary variance in our narrow-age sample who live in the same geographical location; it is possible that the traditional diet pattern derived in this group reflects a slightly worse diet than average, but is not equivalent to the poorer diet found in other, more deprived areas or the general population.

One of the main strengths of this study is the use of comprehensive cognitive testing. Cognitive assessment in several previous studies has been limited to either brief telephoneadministered cognitive tests ${ }^{(19,47)}$, or the $\mathrm{MMSE}^{(14,18,48)}$, which has low sensitivity for detecting age-related cognitive change, or to global cognitive function only ${ }^{(15,17)}$. Furthermore, in the few studies where more than one cognitive outcome has been assessed, often the beneficial effects are inconsistent across domains, or associations with the MMSE were found but not with domain-specific abilities. For instance, in the Three-City cohort ( $n$ 1410, $\geq 65$ years), the Mediterranean diet was associated with slower 5-year MMSE decline, but not with verbal ability, visual memory or episodic memory ${ }^{(9)}$. Wade et al. reported an effect of higher adherence to a Mediterranean diet with 5-year improvements in global cognitive function, visual-spatial organisation and memory, and scanning and tracking, in participants $\geq 70$ years ( $n$ 894), but no associations with verbal memory, working memory or abstract reasoning, in the Maine-Syracuse Longitudinal study ${ }^{(50)}$. The Australian Imaging Biomarkers and Lifestyle study examined an a priori 'AusMeDi' score and two PCA-derived diet patterns (prudent diet and Western diet) in their sample ( $n$ 527, mean 69 years) and reported associations in one domain only (executive function) found in one subgroup (ApoE e4 carriers) ${ }^{(20)}$. In accordance with the current study, which had a similar sample age, they reported no associations with the healthy, 'prudent' PCA dietary pattern. Taken together, the results of studies to date suggest that evidence of the role of different dietary patterns in influencing age-related cognitive decline remains inconclusive and highlight the need for longer follow-up of older people using sensitive cognitive tests which capture variation in age-related cognitive change.

Discrepancies between studies may be the result of cohort differences and methodological variations. First, the lack of associations observed in the current study may partly be due to the relatively homogenous nature of the sample, that is, all are Caucasian, the same age, and living in the same (relatively affluent) area of Scotland. As volunteer participants in a longitudinal study of ageing, they are likely to be more cognitively able than the general population and invested in their cognitive wellbeing. Moreover, those who remained to follow up had significantly higher childhood IQ scores and more education. Therefore, our participants may have a narrower distribution of cognitive function and decline, which could reduce the magnitude of associations in our study.

Second, differences in the measurement of Mediterranean diet adherence make comparisons between studies problematic. Mediterranean diet adherence is traditionally measured using an 'a priori' Mediterranean diet score ${ }^{(42)}$, developed to reflect the traditional diet of those residing near the Mediterranean sea which is rich in vegetables, fish, cereals, fruit, extra-virgin olive oil and legumes, and relatively low in meat and dairy products. Systematic reviews have concluded that studies conducted in Greece and other Mediterranean regions provide strong support for a protective effect of the Mediterranean diet on cognitive decline $^{(6)}$ where nutrient intakes are higher ${ }^{(51)}$. In countries outside of the Mediterranean basin, the use of a pre-defined score can be problematic given that the average diet is unlikely to conform to such ideals. Indeed, the majority of the aforementioned studies reporting null findings are mostly from nonMediterranean samples. Calculation of dietary adherence using patterns derived from whole-diet data, such as in the present study, is more likely to reflect the true nature and complexity of the local Scottish diet, where nutrient intakes are lower than many other countries, and is an approach that other UK studies have used ${ }^{(48,52)}$. Though the current study used an 'a posteriori' measure of Mediterranean-style diet adherence in the main analyses, we also observed null findings using a Mediterranean diet score ${ }^{(42)}$ which may reflect modest adherence to the Mediterranean diet in the Scottish population, as above, and suggest that the measurement method used, is not driving the associations here.

Third, dietary intake in adulthood is strongly associated with early-life cognitive ability - in the LBC1936 (22) and in other samples ${ }^{(53,54)}$ - which makes determining the direction of causation in diet-cognition studies problematic. These studies demonstrate a confounding effect of prior cognitive ability whereby brighter children tend to become brighter adults with healthier lifestyle practices, including dietary choices. Here, marked attenuation was evident when adjustment was made for a measure of participants' cognitive ability at age 11 years, such that intercept associations of diet with visuospatial ability, processing speed and memory, lost significance. This suggests that prior (childhood) cognitive ability level, and other proxy measures such as educational attainment, account for a large part of the variance in diet-cognitive ability associations in later life, and full consideration should be given to such measures in future studies.

There are several strengths and limitations of this study. Strengths of this study include the well-phenotyped sample including detailed dietary data and comprehensive cognitive assessments over five waves of follow-up using multiple tests with proven validity and sensitivity to cognitive deficits. We adjusted for a range of covariates established in the LBC1936, and other studies, as known predictors of cognitive decline and other neurodegenerative disorders, including childhood $\mathrm{IQ}^{(55)}$ and $A p o E$ e4 status $^{(36,56)}$. We used sophisticated statistical methods; our analyses employed a longitudinal design incorporating information collected at each wave into growth curve models enabling detection of linear trends in cognitive change. We excluded those with dementia diagnosed at any point across the five waves of testing, reducing the possibility that predementia states may have affected dietary choices.

One of the limitations of our study is that diet was assessed only at baseline. Baseline diet might not represent a stable diet over the study period. Thus, we were unable to address the relationship between dietary change and cognitive change across the follow-up period. Second, the Mediterranean-style pattern 
identified in the current study contains some (e.g. vegetables, fish, beans), but not all, important aspects of a traditional Mediterranean diet consumed in Greece and other Mediterranean countries. For example, the PCA-derived pattern does not include loadings of $>0.30$ from olive oil, fruits or wine. Third, our sample size at the end of follow-up was reduced due to attrition, though we note that one of the advantages of latent growth curve models is that they have flexibility with regard to missing data due to attrition, as is the case in long-term longitudinal studies. Using full-information strategies, they take into account all measures at each time point regardless of whether participants have complete data throughout the five waves of follow-up. Finally, the LBC1936 are a self-selected sample of relatively well-performing, healthy individuals and as such, our findings may not generalise to other populations.

\section{Conclusion}

In conclusion, we found that greater adherence to a healthy Mediterranean-style diet was associated cross-sectionally with better verbal (crystallised) ability, with the converse being true for a more meat-based traditional diet. However, neither dietary pattern predicted global- nor domain-specific cognitive decline, with the exception of a slightly steeper slope in verbal ability in those with a higher Mediterranean-style diet score at baseline. This association was small, and likely to reflect regression to the mean in higher cognitive ability participants at baseline. In this well-characterised Scottish cohort of older adults, dietary patterns did not significantly influence trajectories of cognitive ageing, beyond known predictors of cognitive change.

\section{Acknowledgements}

The authors would like to thank the LBC research team who collected, entered and checked data used in this manuscript. We thank the staff at the Rowett Research Institute at the University of Aberdeen for FFQ data entry and assistance, and performing dietary pattern analyses. We thank the nursing staff at the Wellcome Trust Clinical Research Facility at the Western General, Edinburgh.

This work was supported by Age UK (Disconnected Mind project); the UK Medical Research Council (MRC; G0701120, G1001245, MR/M013111/1) and a National Institutes of Health (NIH) research grant (I.J.D., R01AG054628). Age UK, MRC and NIH had no role in the design, analysis or writing of this article.

The authors' responsibilities were as follows: J. C. and I. J. D. designed the research; J. C. analysed the data; J. C. interpreted the results and prepared the figures; J. C. drafted the manuscript; J. C. and I. J. D. edited the manuscript; all authors approved the final version of the manuscript.

The authors declare that there are no conflicts of interest.

\section{Supplementary material}

For supplementary material referred to in this article, please visit https://doi.org/10.1017/S0007114520005139

\section{References}

1. Hedden T \& Gabrieli JD (2004) Insights into the ageing mind: a view from cognitive neuroscience. Nat Rev Neurosci 5, 87-96.

2. Tucker-Drob EM, Brandmaier AM \& Lindenberger U (2019) Coupled cognitive changes in adulthood: a meta-analysis. Psychol Bull 145, 273-301.

3. Gardener SL \& Rainey-Smith SR (2018) The role of nutrition in cognitive function and brain ageing in the elderly. Curr Nutr Rep 7, 139-149.

4. Angeloni C, Businaro R \& Vauzour D (2020) The role of diet in preventing and reducing cognitive decline. Curr Opin Psychiatr 33, 432-438.

5. Lourida I, Soni M, Thompson-Coon J, et al. (2013) Mediterranean diet, cognitive function, and dementia: a systematic review. Epidemiology 1, 479-489.

6. Aridi Y, Walker J \& Wright O (2017) The association between the Mediterranean dietary pattern and cognitive health: a systematic review. Nutrients $\mathbf{9}, 674$.

7. Loughrey DG, Lavecchia S, Brennan S, et al. (2017) The impact of the Mediterranean diet on the cognitive functioning of healthy older adults: a systematic review and meta-analysis. Adv Nutr 8, 571-586.

8. van de Rest O, Berendsen AA, Haveman-Nies A, et al. (2015) Dietary patterns, cognitive decline, and dementia: a systematic review. Adv Nutr 6, 154-168.

9. Féart C, Samieri C, Rondeau V, et al. (2009) Adherence to a Mediterranean diet, cognitive decline, and risk of dementia. JAMA 302, 638-648.

10. Galbete C, Toledo E, Toledo JB, et al. (2015) Mediterranean diet and cognitive function: the SUN project. $J$ Nutr Health Aging 19, 305-312.

11. Samieri C, Jutand MA, Féart C, et al. (2008) Dietary patterns derived by hybrid clustering method in older people: association with cognition, mood, and self-rated health. J Am Diet Ass 108, 1461-1471.

12. Scarmeas N, Stern Y, Tang MX, et al. (2006) Mediterranean diet and risk for Alzheimer's disease. Ann Neurol 59, 912-921.

13. Tsai HJ (2015) Dietary patterns and cognitive decline in Taiwanese aged 65 years and older. Int J Geriatr Psychiatr 30, 523-530.

14. Wengreen H, Munger RG, Cutler A, et al. (2013) Prospective study of dietary approaches to stop hypertension-and Mediterranean-style dietary patterns and age-related cognitive change: the Cache County study on memory, health and aging. Am J Clin Nutr 98, 1263-1271.

15. Tangney CC, Kwasny, MJ, Li H, et al. (2011) Adherence to a Mediterranean-type dietary pattern and cognitive decline in a community population. Am J Clin Nutr 93, 601-607.

16. Tangney CC, Li H, Wang Y, et al. (2014) Relation of DASH- and Mediterranean-like dietary patterns to cognitive decline in older persons. Neurology 83, 1410-1416.

17. Cherbuin N \& Anstey KJ (2012) The Mediterranean diet is not related to cognitive change in a large prospective investigation: the PATH Through Life study. Am J Geriatr Psychiatr 20, 635-639.

18. Psaltopoulou T, Kyrozis A, Stathopoulos P, et al. (2008) Diet, physical activity and cognitive impairment among elders: the EPIC-Greece cohort (European Prospective Investigation into Cancer and Nutrition). Public Health Nutr 11, 1054-1062.

19. Samieri C, Okereke OI, Devore E, et al. (2013) Long-term adherence to the Mediterranean diet is associated with overall cognitive status, but not cognitive decline, in women. $J$ Nutr 143, 493-499.

20. Gardener SL, Rainey-Smith SR, Barnes MB, et al. (2015) Dietary patterns and cognitive decline in an Australian study of ageing. Mol Psychiatr 20, 860-866. 
21. Hardman RJ, Kennedy G, Macpherson H, et al. (2016) Adherence to a Mediterranean-Style diet and effects on cognition in adults: a qualitative evaluation and systematic review of longitudinal and prospective trials. Front Nutr 3, 22.

22. Corley J, Starr JM, McNeill G, et al. (2013) Do dietary patterns influence cognitive function in old age? Int Psychogeriatr 25, $1393-1407$.

23. Scottish Council for Research in Education (1949) The Trend of Scottish Intelligence: A Comparison of the 1947 and 1932 Surveys of the Intelligence of Eleven-Year-Old Pupils. London: University of London Press.

24. Taylor AM, Pattie A \& Deary IJ (2018) Cohort profile update: the Lothian Birth cohorts of 1921 and 1936. Int J Epidemiol 47, 1042-1042r

25. University of Aberdeen (2005) Scottish collaborative group food frequency questionnaire. https://www.foodfrequency. org/ (accessed November 2019).

26. Jia X, Craig LC, Aucott LS, et al. (2008) Repeatability and validity of a food frequency questionnaire in free-living older people in relation to cognitive function. $J$ Nutr Health Aging 12, 735-741.

27. Mõttus R, McNeill G, Jia X, et al. (2013) The associations between personality, diet and body mass index in older people. Health Psychol 32, 353-360.

28. Willett, W (2012) Nutritional Epidemiology. Oxford: Oxford University Press.

29. Wechsler D (1998) WAIS-IIIUK Administration and Scoring Manual. London: Psychological Corporation.

30. Wechsler D (1998) WMS-IIIUK Administration and Scoring Manual. London: Psychological Corporation.

31. Deary IJ, Der G \& Ford G (2001) Reaction times and intelligence differences: a population-based cohort study. Intelligence 29, 389-399.

32. Deary IJ, Simonotto E, Meyer M, et al. (2004) The functional anatomy of inspection time: an event-related fMRI study. NeuroImage 22, 1466-1479.

33. Nelson HE \& Willison JR (1991) National Adult Reading Test (NART) Test Manual (Part II). Windsor, England: NFER-Nelson.

34. Holdnack JA (2001) WTAR: Wechsler Test of Adult Reading manual. San Antonio, TX: Psychological Corporation.

35. Lezak MD, Howieson DB, Loring DW, et al. (2004) Neuropsychological Assessment. USA: Oxford University Press.

36. Ritchie SJ, Tucker-Drob EM, Cox SR, et al. (2016) Predictors of ageing-related decline across multiple cognitive functions. Intelligence 59, 115-126.

37. Tucker-Drob EM, Briley DA, Starr JM, et al. (2014) Structure and correlates of cognitive aging in a narrow age cohort. Psychol Aging 29, 236-249.

38. OPCS (1980) Classification of Occupations 1980. London: HMSO.

39. Deary IJ, Whalley LJ, Lemmon H, et al. (2000) The stability of individual differences in mental ability from childhood to old age: Follow-Up of the 1932 Scottish Mental Survey. Intelligence 28, 49-55.
40. Deary, IJ, Whiteman, MC, Starr JM, et al. (2004) The impact of childhood intelligence on later life: following up the Scottish mental surveys of 1932 and 1947. J Pers Soc Psychol 86, $130-147$.

41. Rosseel Y (2012) lavaan: an R package for structural equation modeling. J Stat Software 48, 1-36.

42. Trichopoulou A, Costacou T, Bamia C, et al. (2003) Adherence to a Mediterranean diet and survival in a Greek population. $N$ Engl J Med 348, 2599-2608.

43. Nesselroade JR, Stigler SM \& Baltes PB (1980) Regression toward the mean and the study of change. Psychol Bull 88, 622-637.

44. Salthouse TA (2012) Does the direction and magnitude of cognitive change depend on initial level of ability? Intelligence $\mathbf{4 0}$, 352-361.

45. Li G, Larson EB, Shofer JB, et al. (2017) Cognitive trajectory changes over 20 years before dementia diagnosis: a large cohort study. J Am Geriatr Soc 65, 2627-2633.

46. Salthouse TA (2010) Selective review of cognitive aging. J Int Neuropsychol Soc 16, 754-760.

47. Vercambre MN, Grodstein F, Berr C, et al. (2012) Mediterranean diet and cognitive decline in women with cardiovascular disease or risk factors. J Acad Nutr Diet 12, 816-823.

48. Granic A, Davies K, Adamson A, et al. (2016) Dietary patterns high in red meat, potato, gravy, and butter are associated with poor cognitive functioning but not with rate of cognitive decline in very old adults. J Nutr $\mathbf{4 6}, 265-274$.

49. Radd-Vagenas S, Duffy SL, Naismith SL, et al. (2018) Effect of the Mediterranean diet on cognition and brain morphology and function: a systematic review of randomized controlled trials. Am J Clin Nutr 107, 389-404.

50. Wade AT, Elias MF \& Murphy KJ (2019) Adherence to a Mediterranean diet is associated with cognitive function in an older non-Mediterranean sample: findings from the MaineSyracuse longitudinal study. Nutr Neurosci (epublication ahead of print version 21 August 2019).

51. Castro-Quezada I, Román-Viñas B \& Serra-Majem L (2014) The Mediterranean diet and nutritional adequacy: a review. Nutrients 6, 231-248.

52. Mishra GD, McNaughton SA, Bramwell GD, et al. (2006) Longitudinal changes in dietary patterns during adult life. $\mathrm{Br}$ J Nutr 96, 735-744.

53. Batty GD, Deary IJ, Schoon I, et al. (2007) Childhood mental ability in relation to food intake and physical activity in adulthood: the 1970 British Cohort Study. Pediatrics 19, e38-45.

54. Gale CR, Deary IJ, Schoon I, et al. (2007) IQ in childhood and vegetarianism in adulthood: 1970 British cohort study. BMJ 334, 245.

55. Corley, J, Cox, SR \& Deary, IJ (2018) Healthy cognitive ageing in the Lothian Birth Cohort studies: marginal gains not magic bullet. Psychol Med 48, 187-207.

56. Liu CC, Kanekiyo T, Xu H, et al. (2013) Apolipoprotein E and Alzheimer disease: risk, mechanisms and therapy. Nat Rev Neurol 9, 106-118. 